\title{
The Impact of COVID-19 on Tuberculosis Patients' Behavior of Seeking Medical Care - China, 2020
}

\author{
Yinyin Xia'; Fei Huang'; Hui Chen'; Ni Wang'; Xin Du'; Wei Chen'; Tao Li'; \\ Shitong Huan'; Miaomiao Sun ${ }^{3}$; Jianjun Liu'; Yanlin Zhao ${ }^{1, *}$
}

\section{Summary \\ What is already known on this topic? \\ The coronavirus disease 2019 (COVID-19) pandemic has disrupted the tuberculosis (TB) service system. However, the impact on TB patients in China remains unknown. \\ What is added by this report? \\ This report firstly addressed the impact of COVID-19 on TB patients in China. About half of TB patients did not revisit the hospital due to personal reasons. The reasons for irregular medication and postponing or cancelling examination after full treatment course were different. \\ What are the implications for public health practice? \\ Health education and risk communication should be strengthened for better TB patient management and treatment adherence, especially in light of the COVID- 19 pandemic.}

As a new acute respiratory infectious disease, coronavirus disease 2019 (COVID-19) has become one of the world's most important public health problems. As a high tuberculosis (TB) burden country, China promptly adopted two overarching strategies of containment and suppression in response to the COVID-19 epidemic (1), which have already affected TB control, including notification, follow-up examinations, and treatment outcomes (2-4). However, existing studies presented the impact mainly based on routine surveillance data from the TB service system. The impact on TB patients' behavior of seeking medical care from the perspective of $\mathrm{TB}$ patients remains unknown. To address this issue, China CDC randomly selected 294 counties from 31 provincial-level administrative divisions (PLADs) and conducted a national questionnaire survey in May 2020. The survey result showed that about half of TB patients did not revisit the hospital due to personal reasons, and the reasons for irregular medication and postponing or canceling examination after full treatment course were different. Therefore, health education should be strengthened for better TB patient management in addition to guaranteed uninterrupted service system.

To better understand the potential impact of COVID-19 on TB patients' medical care seeking behavior, we conducted a national TB patients survey. A total of 294 counties were randomly selected $(10 \%$ of all counties) by PLADs, and whether the county had reported cases of COVID-19, of which 116 counties reported COVID-19 cases and 178 counties did not report COVID-19 cases. Then, a total of 18 cases were randomly selected for each county including $6 \mathrm{~TB}$ cases with treatment for less than 2 months, 6 cases treated for 2 months, and 6 cases that finished the whole treatment course from the period of January 25 to April 8, 2020, which was defined as the COVID-19 period as it marked the starting point of the national emergency response to COVID-19 to the reopening of Wuhan city. If there were fewer than 6 cases for any category in the county, then all the $\mathrm{TB}$ cases in that category were surveyed. Finally, a total of 3,224 TB cases were selected and investigated by CDC staff, with $665,1,224$, and 1,335 cases from each group, respectively. In the questionnaire, some questions were for all cases and some questions were for specific TB cases (Table 1).

Of the $850 \mathrm{~TB}$ cases that were diagnosed in the COVID-19 period, the diagnoses of $192(22.6 \%)$ cases were affected. There were $81(42.2 \%), 52(27.1 \%)$, and $59(30.7 \%)$ cases who postponed seeking medical care due to traffic restrictions, TB service disruptions, and personal reasons, respectively. No significant differences existed between counties with/without COVID-19 in terms of the diagnosis of TB $(P=0.597)$ and the main underlying reason for postponing seeking care $(P=0.231)$.

Of the 3,224 TB cases that should have taken antiTB drugs regularly in the COVID-19 period, only 110 $(3.4 \%)$ cases reported irregular intake of medication 
TABLE 1. Main questions surveyed for different groups of tuberculosis (TB) cases conducted by China CDC in China May, 2020.

\begin{tabular}{|c|c|c|c|}
\hline Questions & $\begin{array}{c}\text { Treated less } \\
\text { than } 2 \\
\text { months } \\
\end{array}$ & $\begin{array}{c}\text { Treated } \\
\text { for } 2 \\
\text { months } \\
\end{array}$ & $\begin{array}{l}\text { Finished } \\
\text { treatment }\end{array}$ \\
\hline If diagnosis of TB was affected and the main underlying reason & Yes & Yes & No \\
\hline If regular intake of medication was affected and the main underlying reason & Yes & Yes & Yes \\
\hline If sputum examination after 2 months' treatment was affected and the main underlying reason & No & Yes & No \\
\hline If sputum examination after full treatment course was affected and the main underlying reason & No & No & Yes \\
\hline
\end{tabular}

and no significant differences existed between counties with/without COVID-19 $(P=0.618)$. Due to side effects and personal reasons, $48(43.6 \%)$ and 62 $(56.4 \%)$ cases, respectively, did not take medication regularly. There were significant differences between counties with COVID-19 and without COVID-19 in terms of the main underlying reason for irregular medication $(P<0.001)$.

Of the 1,224 TB cases that should have sputum examination after 2 months' treatment in the intensive period, $322(26.3 \%)$ cases were affected. A total of 110 $(34.2 \%), \quad 51 \quad(15.8 \%)$, and $161(50.0 \%)$ cases postponed or canceled the examination due to traffic restrictions, TB service disruptions, and personal reasons, respectively. There were no significant differences between counties with COVID-19 and without COVID-19 in terms of the percentage of sputum examination $(P=0.794)$ and the main underlying reason $(P=0.454)$.

Of the $1,335 \mathrm{~TB}$ cases that should have sputum examination after full treatment course in the intensive period, $379(28.4 \%)$ cases were affected, and there was no significant difference between counties with COVID-19 and without COVID-19 $(P=0.794)$. A total of $129(34.0 \%), 41(10.8 \%)$, and $209(55.2 \%)$ cases postponed or canceled the examination due to traffic restrictions, TB service disruptions, and personal reasons, respectively. There were significant differences between counties with COVID-19 and without COVID-19 in terms of the main underlying reason $(P=0.002)$ (Table 2$)$.

\section{DISCUSSION}

The COVID-19 epidemic in China has greatly affected the behavior of TB patients' seeking medical care, but there was no significant difference between counties with or without a COVID-19 epidemic. About a quarter of TB patients had reported that TB diagnoses and follow-up examinations were affected by the COVID-19 epidemic, which was much higher than the proportion of $\mathrm{TB}$ patients for whom regular intake of medication was affected. About half of TB patients did not revisit the hospital for follow-up examinations. In terms of diagnostic delays, the main underlying reasons were traffic restrictions, followed by personal reasons and TB service disruptions. The reasons for irregular intake of medication and postponing or canceling examinations after a full treatment course were different between counties with/without COVID-19 epidemic.

The behaviors of TB patients seeking medical care were all affected by the COVID-19 epidemic, which was similar to previous studies from China $(4-5)$. However, regular intake of medication was the least affected. The main reason was that TB patients did not necessarily need to go out to visit the hospital for antiTB drugs during the COVID-19 period. Under technical guidance of China CDC, healthcare workers across the country tried their best to solve the supply of anti-TB drugs and deliver the drugs to patients by different methods.

The results from our study presented no significant differences between counties with/without COVID-19 regarding the behavior of $\mathrm{TB}$ patients seeking medical care. As we knew little about the new infectious pathogen at the beginning of the COVID-19 outbreak, China initiated the nationwide emergency response on January 25, 2020 to tackle COVID-19 and implemented a series of nonpharmaceutical public health interventions and enforced them strictly across the country $(1,6-7)$. The percentage of traffic restrictions resulting in diagnostic delays and postponing or cancelling examinations after full treatment course was a little higher in COVID-19 counties than non-COVID-19 counties, which indicated that traffic maybe was more strictly restricted in COVID-19 counties. Personal reasons, including fear of infection with COVID-19, objection of family members, and feeling lack of necessity, still accounted for the majority of TB patients who canceled or postponed follow-up examinations, which was similar 
TABLE 2. The questionnaire results of tuberculosis (TB) cases from randomly selected counties conducted by China CDC in China - May, 2020.

\begin{tabular}{|c|c|c|c|c|}
\hline \multirow{2}{*}{ Type } & \multirow{2}{*}{ Total } & \multicolumn{2}{|c|}{ Surveyed counties } & \multirow{2}{*}{$P$-value } \\
\hline & & No COVID-19 & COVID-19 & \\
\hline Diagnosis of TB & & & & 0.597 \\
\hline Not affected & $658(77.4)$ & $267(76.5)$ & $391(78.0)$ & \\
\hline Affected & $192(22.6)$ & $82(23.5)$ & $110(22.0)$ & \\
\hline Reason of diagnosis delay & & & & 0.231 \\
\hline Traffic restriction & $81(42.2)$ & $29(35.4)$ & $52(47.3)$ & \\
\hline TB service disruption & $52(27.1)$ & $26(31.7)$ & $26(23.6)$ & \\
\hline Personal reason & $59(30.7)$ & $27(32.9)$ & $32(29.1)$ & \\
\hline Regular intake of medication & & & & 0.618 \\
\hline Not affected & $3,114(96.6)$ & $1,177(96.8)$ & $1,937(96.5)$ & \\
\hline Affected & $110(3.4)$ & $39(3.2)$ & $71(3.5)$ & \\
\hline Reason of irregular medication & & & & $<0.001$ \\
\hline Side effect & $48(43.6)$ & $26(66.7)$ & $22(31.0)$ & \\
\hline Personal reason & $62(56.4)$ & $13(33.3)$ & $49(69.0)$ & \\
\hline \multicolumn{3}{|c|}{ Sputum examination after 2 months' treatment } & & 0.794 \\
\hline Yes & $902(73.7)$ & $326(73.3)$ & $576(73.9)$ & \\
\hline No & $322(26.3)$ & $119(26.7)$ & $203(26.1)$ & \\
\hline \multicolumn{3}{|c|}{ Reason of postponing or cancelling examination after 2 months' treatment } & & 0.454 \\
\hline Traffic restriction & $110(34.2)$ & $41(34.5)$ & $69(34.0)$ & \\
\hline TB service disruption & $51(15.8)$ & $15(12.6)$ & $36(17.7)$ & \\
\hline Personal reason & $161(50.0)$ & $63(52.9)$ & $98(48.3)$ & \\
\hline \multicolumn{3}{|c|}{ Sputum examination after full treatment course } & & 0.794 \\
\hline Yes & $956(71.6)$ & $363(72.0)$ & $593(71.4)$ & \\
\hline No & $379(28.4)$ & $141(28.0)$ & $238(28.6)$ & \\
\hline \multicolumn{3}{|c|}{ Reason of postponing or cancelling examination after full treatment course } & & 0.002 \\
\hline Traffic restriction & $129(34.0)$ & $33(23.4)$ & $96(40.3)$ & \\
\hline TB service disruption & $41(10.8)$ & $15(10.6)$ & $26(10.9)$ & \\
\hline Personal reason & $209(55.2)$ & $93(66.0)$ & $116(48.8)$ & \\
\hline
\end{tabular}

Note: Data are presented as $\mathrm{n}(\%)$ unless otherwise stated.

to the impact of Ebola on TB control (8). This also implies the importance of health education and risk communication with TB patients, which could help $\mathrm{TB}$ patients better understand the reasoning for why they should treat $\mathrm{TB}$ regularly and how to avoid infection with COVID-19 when they revisit the hospital. This will help improve TB patient management and treatment adherence.

This study was subject to several limitations. First, all the impacts were investigated from $\mathrm{TB}$ cases who were already diagnosed by TB designated hospitals, and we did not know the exact impact of COVID-19 on these presumptive TB cases who did not go to the hospital for a doctor. Second, we only analyzed the short-term impact of COVID-19 on TB patients' behavior, while the long-term impact on behavior of seeking medical care is still unknown. The other limitations included recall bias due to the retrospective surveys and lack of detailed information for TB cases to identify high-risk groups who suffered most during the COVID-19 epidemic.

In conclusion, the COVID-19 epidemic in China has imposed a substantial impact on the behavior of TB patients' seeking medical care, and for TB patients who did not revisit the hospital, half of them were due to personal reasons. Health education and risk communication with $\mathrm{TB}$ cases should be strengthened for better TB patient management and treatment 
adherence in addition to guaranteed uninterrupted service systems.

Funding: The National Health Commission of China-Bill \& Melinda Gates Foundation TB Collaboration Project (OPP1137180).

doi: $10.46234 / \mathrm{ccdcw} 2021.143$

\# Corresponding author: Zhao Yanlin, zhaoyl@chinacdc.cn.

${ }^{1}$ Chinese Center for Disease Control and Prevention, Beijing, China;

${ }^{2}$ China office, The Bill \& Melinda Gates Foundation, Beijing, China;

${ }^{3}$ China office, PATH, Beijing, China.

Submitted: May 07, 2021; Accepted: June 23, 2021

\section{REFERENCES}

1. Li ZJ, Chen QL, Feng LZ, Rodewald L, Xia YY, Yu HL, et al. Active case finding with case management: the key to tackling the COVID-19 pandemic. Lancet 2020;396(10243):63 - 70. http://dx.doi.org/10.1016/ S0140-6736(20)31278-2.

2. Chen HG, Zhang KL. Insight into the impact of the COVID-19 epidemic on tuberculosis burden in China. Eur Respir J 2020; 56(3):2002710. http://dx.doi.org/10.1183/13993003.02710-2020.

3. Huang F, Xia YY, Chen H, Wang N, Du X, Chen W, et al. The impact of the COVID-19 epidemic on tuberculosis control in China. Lancet Reg Health: West Pac 2020;3:100032. http://dx.doi.org/10.1016/j. lanwpc.2020.100032.

4. Wu Z, Chen J, Xia Z, Pan Q, Yuan Z, Zhang W, et al. Impact of the COVID-19 pandemic on the detection of TB in Shanghai, China. Int J Tuberc Lung Dis 2020;24(10):1122 - 4. http://dx.doi.org/10.5588/ ijtld.20.0539.

5. Liu Q, Lu P, Shen Y, Li CW, Wang JM, Zhu LM, et al. Collateral impact of the coronavirus disease 2019 (COVID-19) pandemic on tuberculosis control in Jiangsu province, China. Clin Infect Dis 2020ciaa1289. http://dx.doi.org/10.1093/cid/ciaa1289.

6. Pan A, Liu L, Wang CL, Guo H, Hao XJ, Wang Q, et al. Association of public health interventions with the epidemiology of the COVID-19 outbreak in Wuhan, China. JAMA 2020;323(19):1915 - 23. http://dx. doi.org/10.1001/jama.2020.6130.

7. Tian HY, Liu YH, Li YD, Wu CH, Chen B, Kraemer MUG, et al. An investigation of transmission control measures during the first 50 days of the COVID-19 epidemic in China. Science 2020;368(6491):638 - 42. http://dx.doi.org/10.1126/science.abb6105.

8. Desta KT, Kessely DB, Daboi JG. Evaluation of the performance of the National Tuberculosis Program of Liberia during the 2014-2015 Ebola outbreak. BMC Public Health 2019;19(1):1221. http://dx.doi.org/10 1186/s12889-019-7574-7. 